\title{
Generating a train rescheduling timetable considering the train routes on tracks and car types
}

\author{
T. Katori, H. Yamazaki \& T. Izumi \\ Nihon University, Japan
}

\begin{abstract}
It is an important subject to automatically generate train rescheduling timetables after a transport disorder has occurred. In urban areas in Japan, for the convenience of passengers, JR and private companies have lines that converge with each other. However, certain types of cars cannot run on other companies' tracks and this may hamper rescheduling operations. Therefore, we propose an automatic method to generate train rescheduling timetables. For the through operation and turning operation, we consider routes on tracks and different car types. We treat train rescheduling as returning trains to their original location in rescheduling time. Generating a rescheduling timetable is an operation that results in trains returning to their original location in rescheduling time. For this method, we use a recursive tree structure for the route on track, and calculate the schedule by round robin. All solutions can become options for rescheduling. Choosing from the several options that are calculated, our method adopts the solution that has the even distribution of headway at the main stations.

Keywords: train rescheduling timetable, tree structure, recursive processing, car types, train routes.
\end{abstract}

\section{Introduction}

Safety and punctuality are characteristics of a railway system, but sometimes traffic accidents, equipment failure or disasters cause transport disorder, and accordingly the train schedule will differ from the original timetable.

Train rescheduling is a transitional situation to return to the original schedule. The rescheduling timetable is generated by expert operators, but due to the shortage of experts, attempts are made to generate rescheduling plans by 
computer [1]. Nowadays, improved computer performance and the inclusion of train schedules in train operating and management systems [2] have made it possible to automatically generate timetables under limited conditions [3]. We are studying a way to automatically generate train rescheduling timetables, which enable trains to return to their original location after the disorder. In this paper, we propose an algorithm to generate rescheduling timetables considering routes on tracks and car types [4]. Turning and passing other trains at the station are considered by routes on track. Trains running through to other companies' tracks are considered by car type.

\section{Methodology of the rescheduling}

\subsection{Problems of train rescheduling}

Trains will not be allowed to run under the following two conditions:

- At stations where turning points are not available.

- If the car types are different from other companies, only certain car types will be able to run through to the other companies' tracks.

Other cases can, for example, include the impossibility of preparing a train crew. Therefore, train rescheduling has to take into account tracks, equipment, car types and so on.

There are many solutions possible to reschedule the timetable. In the field of computer science, this problem is called an explosion of combinations.

To get effective solutions, the search space needs to be limited, and a suitable evaluation function needs to be established.

It is difficult to generate and evaluate a train rescheduling timetable that takes into account detailed conditions, because there are many influencing elements. Therefore, to generate automatic train rescheduling that is suitable for all situations is impossible and generally a reliable and good solution cannot be obtained [3].

\subsection{Generation of train rescheduling timetables considering routes on track and car types}

To solve the above problems, we propose a method to generate rescheduling timetables considering the routes on track and car types. If the routes on tracks are considered, it becomes possible to judge existing points to turn or to pass.

In this study, train rescheduling is defined as an operation to return trains from a different location to the location where they ought to be in due time. This is especially effective for lines where different car types are running intermingled.

In the above case, it is not always necessary to return trains to their original position. At times it suffices to return them to a location where trains with the same car types run. This increases rescheduling possibilities as well as shortens the time to finish the rescheduling process. Similar to block on track, a track 
layout is divided by unit time, and a block includes one train only. Therefore, the tracks are dealt with in a digital model, and the search space becomes small.

In order to realize operation resumption based on the rescheduling, crew planning is also crucial. However, crew planning is out of the scope of this research.

\subsection{Research assumptions}

We assume the following:

- $\quad$ The timetable unit is $30 \mathrm{sec}$.

- Minimum train gap time is $5 \mathrm{~min}$.

- Minimum stopping time is $5 \mathrm{~min}$ at turning stations, but $30 \mathrm{sec}$ at other stations.

- Running time between stations is the same for rescheduling as for the normal schedule.

- Only local trains are under consideration.

- $\quad$ All trains have the same driving performance.

- $\quad$ Trains do not merge or divide during operation.

Based on the above assumptions, we deal with a simple model.

\section{Track distribution and timetable data representation}

\subsection{Track distribution and timetable}

Routes are determined by tracks. Tracks are connected to each other. The direction in which a train is moving is determined. Therefore, it becomes possible to trace the routes that trains can possibly use.

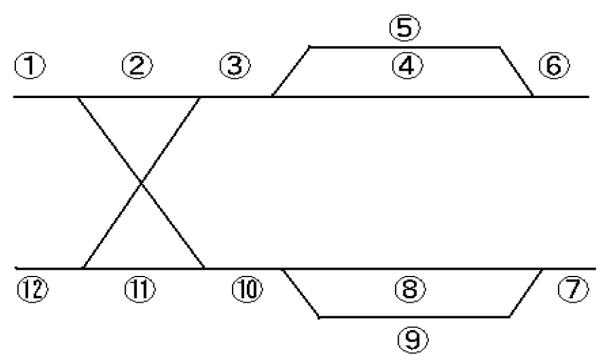

Figure 1: Track layout example.

Accordingly, tracks are divided by unit time (block) and each block is given a node ID number. By expressing these relations in a directed graph, the tracks are easily recognized by the numbers.

Figure 1 shows an example of a track model, and Table 1 displays the data contents for Figure 1. 
Table 1: Contents of array for track layout in Figure 1.

\begin{tabular}{|c|c|}
\hline Location(block) & Connecting block \\
\hline 1 & 2 \\
\hline 2 & 3 \\
\hline 3 & 4 \\
\hline 3 & 5 \\
\hline 4 & 6 \\
\hline$:$ & $:$ \\
\hline 11 & 12 \\
\hline
\end{tabular}

In Table 1, block No. 1 and 2 are connected. This means that if a train is positioned on block No. 1, the train can move to block No. 2. The train on block No. 3 can also run to block no. 4 or 5 .

This data structure can represent the possibility of moving from one block to another considering the connection of tracks.

Strong characteristics of this data structure are the possibility of managing in detail the train location on the tracks, and to add or expand data easily.

Also, at the time of rescheduling, the data structure can arrange running limitations for different car types, and disconnect tracks.

\subsection{Grouping of routes on tracks}

A train route is limited by its track layout. The direction in which the train will move is also decided. To shorten computation time, tracks are grouped to a set by turning equipment or switching devices, and the routes are calculated by the grouped sets. These group data are, similar to the track data structure, composed with connected data of the neighbouring group.

The routes that are calculated by unit time can be integrated by grouping several tracks. For the track layout of Figure 1, eight route groups are possible.

$$
\begin{aligned}
\mathrm{G} 1 & =\{1,2,3,4,6\} \\
\mathrm{G} 2 & =\{1,2,3,5,6\} \\
\mathrm{G} 3 & =\{7,8,10,11,2,1\} \\
\mathrm{G} 4 & =\{7,9,10,11,2,1\} \\
\mathrm{G} 5 & =\{7,8,10,11,12\} \\
\mathrm{G} 6 & =\{7,9,10,11,12\} \\
\mathrm{G} 7 & =\{12,11,2,3,4,6\} \\
\mathrm{G} 8 & =\{12,11,2,3,5,6\}
\end{aligned}
$$

Figure 2 shows some of the track or route groups. A train can move one block per unit time in between broken lines. A pointer is a route that is composed with some blocks. The groups can also express unit time. If any train is located in the same group, it is possible to check train crashes by using the route data per unit time. 


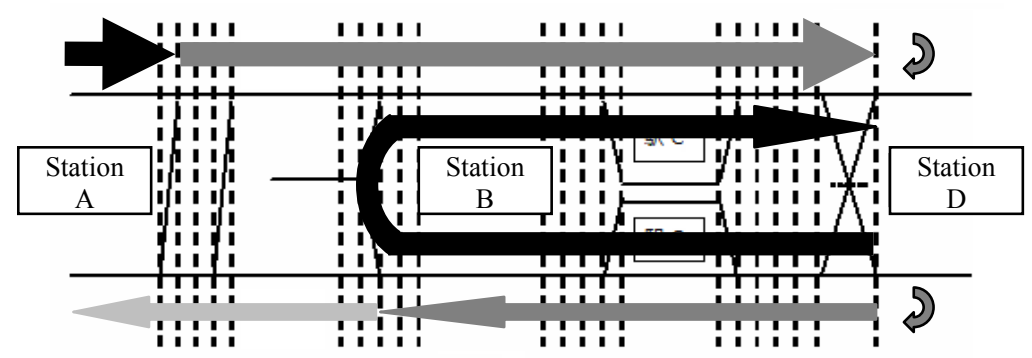

Figure 2: Grouping for track layout in Figure 1.

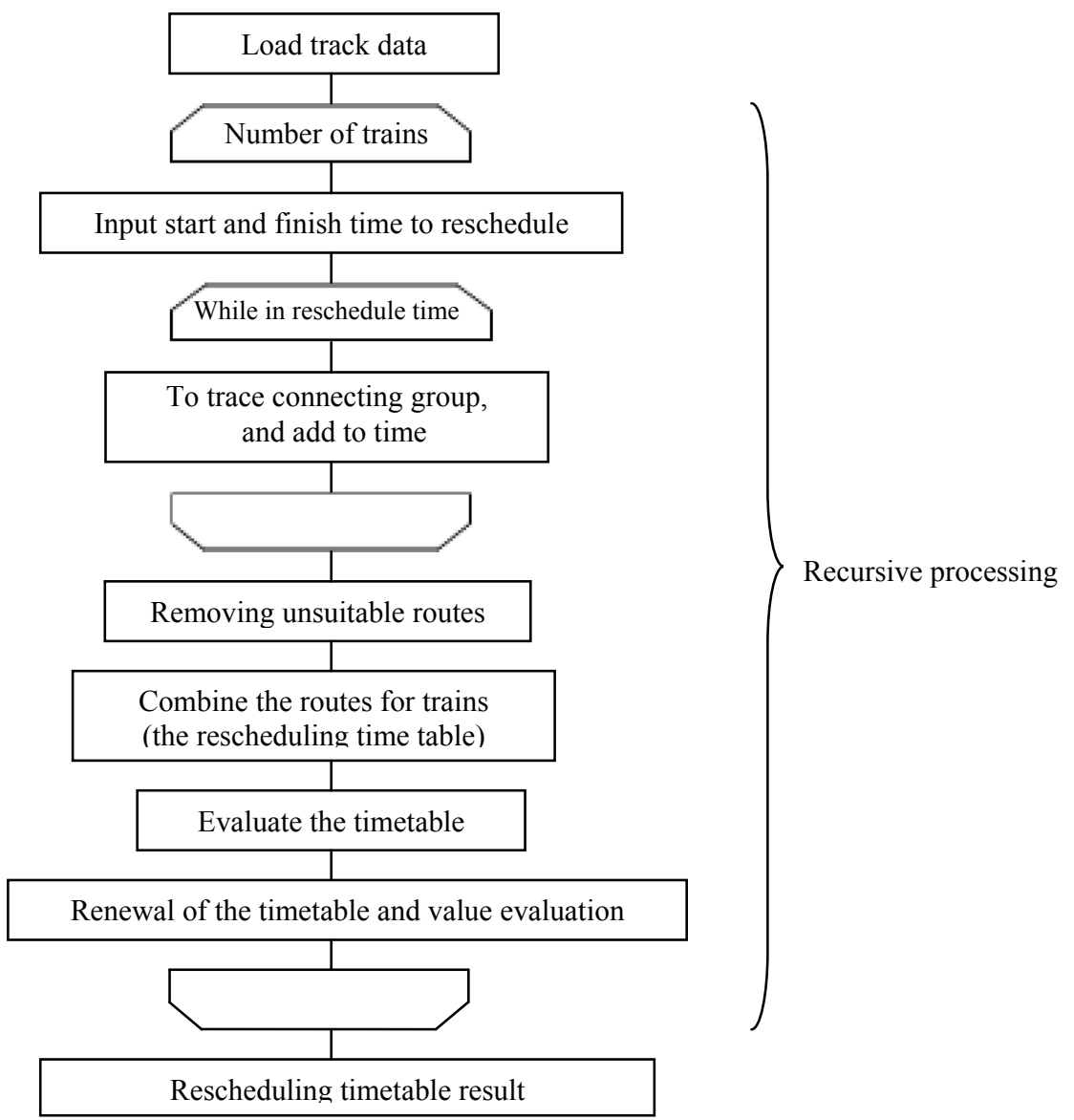

Figure 3: Rescheduling timetable generation flowchart. 


\section{Generating a train rescheduling timetable}

\subsection{Outline to generate a train rescheduling timetable}

Figure 3 shows a flowchart to generate a train rescheduling timetable. First, track layout data are inputted. Start and finish time and train location are also inputted.

A group adjoining the subject point becomes the next route and subject point. The routes are searched by recursive processing. This procedure is repeated for the number of trains necessary.

Solutions of the calculation are expressed by the routes' tree, the root being the starting location, and a leaf being a terminal. At the same time, it is not allowed for the trains to be located in the same block. The solutions are calculated by round robin.

At the finish time, a car type can return to locations with cars of the same type, considering the leaf number of the tree.

The combinations of these trees are solutions, but same locations of different train are not permitted at the same time, because train crashes have to be avoided.

\subsection{Data input}

Track's data that are expressed in Table 1 (connection of groups and relation of group and block) are inputted. Rescheduling start and finish time, as well as train location are inputted for computer.

\subsection{Generation of routes}

This procedure is the most important for this method. It uses the tree data structure for recursive processing.

In the tracks groups are prepared in procedure 4.2, and the adjoining group becomes the next route.

This procedure is repeated until the rescheduling finish time by recursive processing, and the routes develop the tree structure. If there are any switches, the routes have branches.

Figure 4 shows an example of the tree developed for part of track layout in Figure 1.

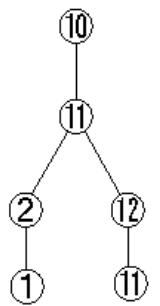

Figure 4: An example of tree structure of the routes. 
In Figure 1, a train moves in the left direction. The train at No. 10 can run to No. 11 by the connected relation in Table 1. Also, the train can move to No. 12 and No. 2 from No. 11, which is expressed in the route tree of Figure 4. However, the leaf number on these routes must be the original location at the finish time. The route trees are made up for the necessary number of trains, and the route combinations are solution options for the rescheduling timetable.

The tree's height indicates the rescheduling time necessary from start to finish. All routes are searched by round robin, but computation time increases.

\subsection{Removing non-suitable routes}

At the finish time, a leaf of the tree must be the original location. The routes satisfying the conditions are stored. These procedures are repeated for the necessary number of trains.

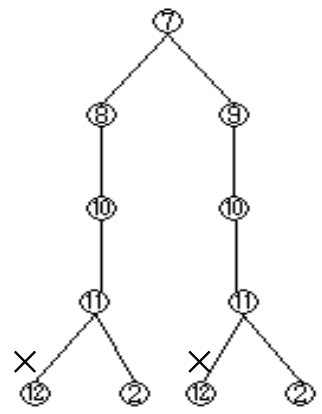

Figure 5: Removing non-suitable routes.

Figure 5 shows an example of removing non-suitable routes for track layout in Figure 1. In Figure 5, if a train is located in No. 7 in Figure 1, the train must be in No. 2 at the finish time. But this tree includes a leaf No. 12. Therefore, the branch between No. 11 and 12 is removed.

\subsection{Combination of routes by trains}

On the train routes, locations at the same time are compared with each other. Routes that do not interfere with one another are combined. The rescheduling timetable is generated. If there are any trains at the same time and location, then this means they hinder each other, and the combination is not adopted.

Figure 6 is an example of two routes that have same location at the same moment, and accordingly this combination is not adopted. 


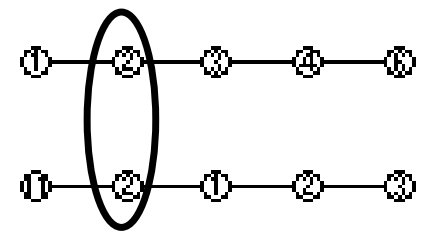

Figure 6: Route interference example.

\subsection{Evaluation for train rescheduling timetable}

After the removal, there are still many options of train rescheduling. These options are evaluated by even headway time at the main stations.

The evaluation value is given the following expression (2),

$$
\mathrm{E}=\Sigma \sum\left(\mathrm{x}_{\mathrm{ij}}-\mathrm{x}_{\mathrm{iave}}\right)^{2}
$$

where:

E: evaluation value;

$\mathrm{x}_{\mathrm{ij}}$ : headway time at main station No. $\mathrm{i}$ between train $\mathrm{j}-1$ and train $\mathrm{j}$;

$\mathrm{x}_{\mathrm{iave}}$ : average time of headway at main station No. i;

i: main station number.

The solution of minimum value is adopted by this evaluation expression.

\section{Some examples of rescheduling and discussion}

The proposed method of generating a train rescheduling timetable is applied to a sample track.

\subsection{Applied model and conditions}

The track layout is modelled on the Tokyo Metro Tozai line and Toyo Rapid line. Figure 7 shows the track layout, and Table 2 indicates the size and conditions of the track.

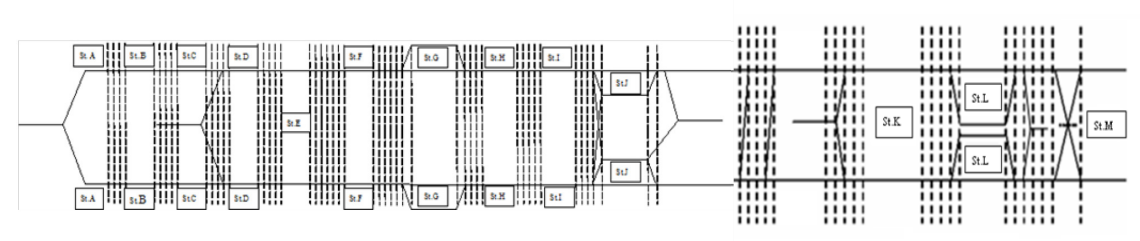

Figure 7: Applied track layout (model for Tozai and Toyo Rapid line in Tokyo). 
Table 2: Size and conditions of the sample track layout.

\begin{tabular}{|c|c|}
\hline Number of stations & 13 \\
\hline One-way running time & 43 min. \\
\hline Number of turning facilities & 4 stations \\
\hline
\end{tabular}

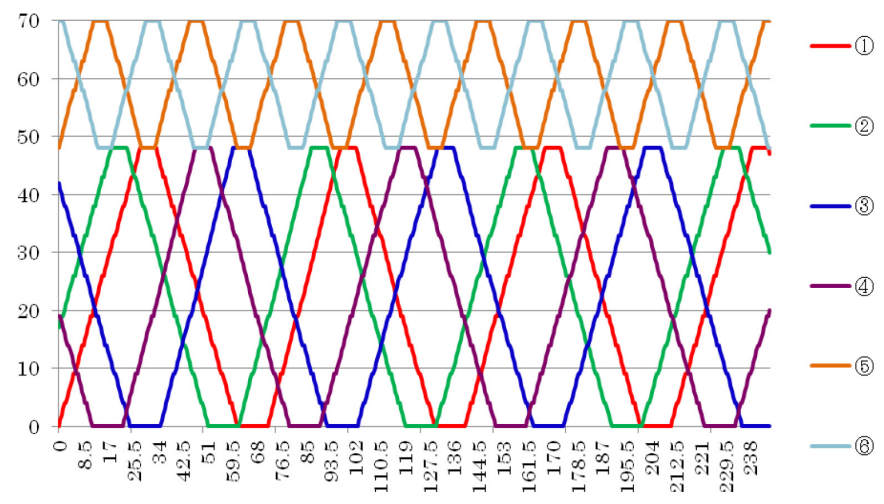

Figure 8: An original timetable for the sample track.

Figure 8 is an original diagram (timetable) for this track.

The setting conditions of transport disorder are as follows:

1) Three minutes after the accident, the trains receive the information from the operator.

2) All trains stop for 30 minutes after the accident.

3) All trains have the same driving performance. If a train runs between stations at the accident, the train runs until the next station only.

In 150 minutes for six trains, the train rescheduling was generated.

\subsection{Some examples of a train rescheduling timetable}

(1) Restoration for all tracks at the same time.

Figure 9 shows an example of a generated diagram. Dark masking means all stopping time zones, and light means rescheduled operation zones (150 minutes). Solid lines indicate the timetable of the original plan. When solid lines and broken lines superimpose, the train returns to the same car type location.

The options for rescheduling timetables include many non-realized plans. To calculate a realized solution, this method needs many other evaluation values.

(2) The track between Station A and B remains closed for another 30 minutes.

Figure 10 shows another rescheduling diagram under more complex conditions. For these conditions, this method can solve and generate the train diagram (timetable). 


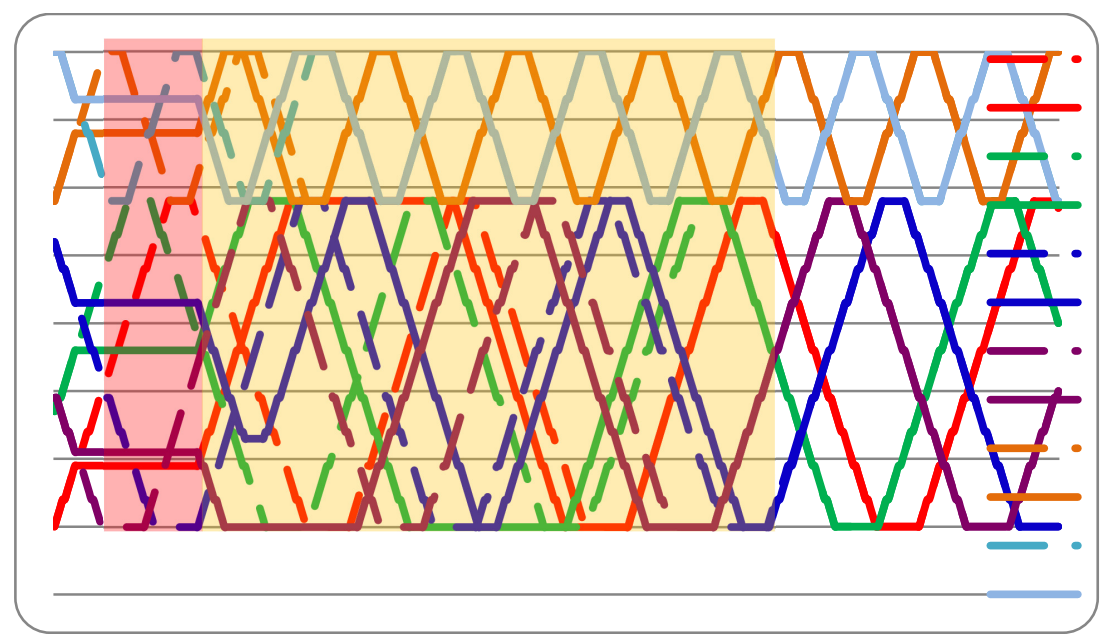

Figure 9: An example of a generated rescheduling timetable.

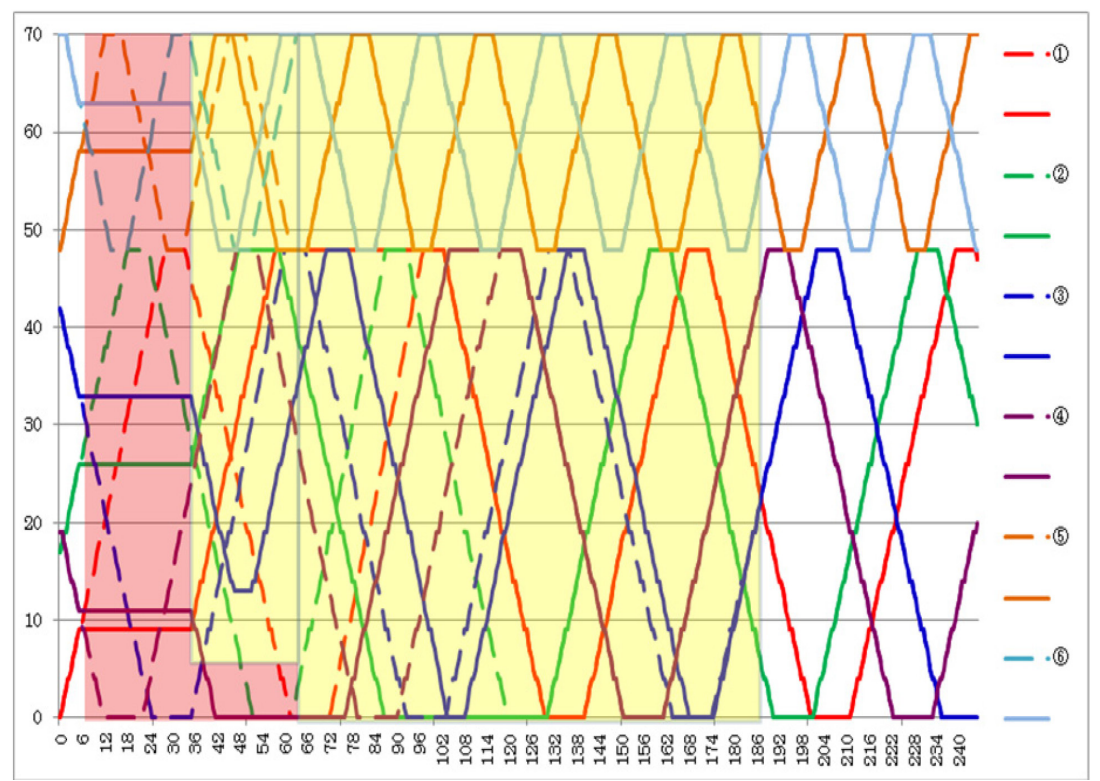

Figure 10: Another example of a rescheduling timetable under other conditions.

\subsection{Discussion}

By this proposed method, to shorten computation time, we adopted track groups. It is easy to change track data. The train moving time is the tree's height in the 
graph data. Therefore, it is possible to consider running section limitation per car types, and rapid train operation.

The route searching is expressed by tree data structure, and all solutions become options. On the other hand, an increasing number of trains or disorder on a large scale cause a long computation time.

For timetable generation, sometimes the timetable needs to be re-generated because of sudden varied conditions, so the computation time should be shorter.

\section{Conclusion}

To apply to turning or through running trains, we propose generating a train rescheduling timetable considering route on track and car types. Generating a timetable is considered as a task to return trains to original locations in provided time. Trains return to the location where there are the same car types.

Track and routes are expressed by graph data and tree structure. Accordingly, no route options are overlooked. The evaluation value is even distribution of headway time at the main stations.

In future research, we will include different car types, to remove non-realised timetables, and to shorten computation time, among others.

\section{Acknowledgement}

We thank Assistant Professor Ruth Vanbaelen, Nihon University, for English language assistance.

\section{References}

[1] T. Nakamura, K. Ihara, "The present conditions and subjects for operation and management system of railway", The Journal of The Institute of Electrical Engineers of Japan, Vol. 124, No. 5, pp. 279-283 (May/2004) (in Japanese).

[2] The Institute of Electrical Engineers of Japan, "Latest Technologies of Electric Railway", Corona Publishing (Sep/2000) (in Japanese).

[3] The Institute of Electrical Engineers of Japan, "The Technologies of Recovering for Railway Timetable", Ohm-sha (Aug/2010) (in Japanese).

[4] Hiroya Yamazaki, Teruomi Katori, Takashi Izumi, "To generate a train rescheduling timetable considering trains routes on tracks and rolling stocks", The Papers of Technical Meeting on Transport and Electric Railway, IEE Japan, TER-12-52, pp. 29-31 (Oct/2012) (in Japanese). 